\title{
Modification of polylactic acid surface using RF plasma discharge with sputter deposition of a hydroxyapatite target for increased biocompatibility
}

S.I. Tverdokhlebov ${ }^{1}$, E.N. Bolbasov ${ }^{1}$, E.V. Shesterikov ${ }^{1}$, L.V. Antonova², A. S. Golovkin², V. G. Matveeva $^{2}$, D.G. Petlin ${ }^{3}$, Y.G. Anissimov ${ }^{3}$

1. Tomsk Polytechnic University, 30 Lenin Avenue, Tomsk 634050, Russian Federation

2. Federal State Budgetary Institution Research Institute for Complex Issues of Cardiovascular Disease, 6 Sosnovy Blvd, Kemerovo 650002, Russian Federation

3. Griffith University, School of Natural Sciences, Engineering Dr., Southport, QLD 4222, Australia

Corresponding author: Sergei I. Tverdokhlebov, e-mail: tverd@tpu.ru, phone: +7(3822)56-3437, Tomsk Polytechnic University, 30 Lenin Avenue, Tomsk 634050, Russian Federation

\begin{abstract}
Surface modification of polylactic acid (PLLA) by plasma of radio-frequency magnetron discharge with hydroxyapatite target sputtering was investigated. Increased biocompatibility was demonstrated using studies with bone marrow multipotent mesenchymal stromal cells. Atomic force microscopy demonstrates that the plasma treatment modifies the surface morphology of PLLA to produce rougher surface. Infrared spectroscopy and X-ray diffraction revealed that changes in the surface morphology are caused by the processes of PLLA crystallization. Fluorescent X-ray spectroscopy showed that the plasma treatment also changes the chemical composition of PLLA, enriching it with ions of the sputtered target: calcium, phosphorus and oxygen. It is hypothesised that these surface modifications increase biocompatibility of PLLA without increasing toxicity.
\end{abstract}


Keywords: polylactic acid, biomaterial, biocompatibility, magnetron sputter deposition, calcium-phosphate

\section{Introduction}

A biomaterial is a material intended to interface with biological systems to treat, enhance or replace any tissue, organ or function of the body [1]. Currently polylactic acid (PLLA) is one of the most widely used biomaterials [2].

PLLA is a polymer with the degree of crystallinity dependent on the molecular weight and polymer treatment parameters. PLLA is biocompatible and degrades into non-toxic components with a well-described degradation rate in vivo and has been used as degradable surgical sutures for a long time. It has gained US Food and Drug Administration approval for clinical use [3].

The surface properties of polymer materials play a crucial role in determining the overall biocompatibility, since the surface of materials comes first in contact with biological environment [4]. Surface morphology and its physiochemical properties have a major influence on the attachment of cells, they determine cell topology, spatial orientation of cell's cytoskeleton components and many other important parameters [5-7].

PLLA is chemically inert and has no reactive side-chain groups, which makes it challenging to modify its surface and bulk. PLLA is comparatively hydrophobic, with a static water contact angle of about $80^{\circ}$. This leads to low cell affinity and can provoke an inflammatory response from the living host upon direct contact with biological fluids [8, 9].

Non-thermal plasma treatments (plasma corona discharge, dielectric barrier discharge, etc.) are often used for inserting chemically-reactive functional groups on polymeric substrates to increase the biocompatibility [10]. While non-thermal plasma surface treatments are preferred for simplicity, modification of PLLA under thermal plasma conditions remain less popular owing to inherent difficulties associated with identifying appropriate plasma conditions and complimenting target material(s) for a specific (bio)-material surface treatment [11].

Radio-frequency magnetron sputtering (RFMS) of a solid dielectric target is a common way to create coatings with high biocompatibility on metal surfaces and biostable polymeric materials such as polyethylene, silicone and polytetrafluoroethylene [12-18]. The RFMS method is based on the sputtering of material in vacuum due to the bombardment of the target surface with the working gas ions which are formed in the abnormal glow discharge plasma when a magnetic field is applied. Thus, the application of the RFMS method allows modifying the plasma 
composition in a wide range not only by changing the atmosphere in the vacuum chamber, but also by changing the chemical composition of the sputtered target [19-23], which opens up new possibilities for the modification of PLLA surface.

Until now only few papers investigated the PLLA surface modification by using RFMS method $[24,25]$. Such situation limits the application of the RFMS method as a universal technique for modifying various types of polymeric materials and narrows the range of possible methods of surface modification of biodegradable polymers for biomedical applications.

We have previously shown that the application of this method for modifying PLLA surface allows increasing the free energy and the biocompatibility of the surface [26]. In the present paper we continue the study of the effect of the RFMS modification on biocompatibility and chemical composition of the treated films. The mechanisms of the formation of highly rough surface during the plasma treatment were further investigated.

\section{Materials and methods}

Polymer films were prepared from a $4 \%$ solution of the polymeric material (Poly (L-lactide) PURASORB $^{\circledR}$ PL 38, Purac) in dichloromethane $\left(\mathrm{CH}_{2} \mathrm{Cl}_{2}\right.$, Panreac Química S.L.U.). The polymer solution of $12( \pm 1)$ g was placed in a specially designed glass bath with a polished bottom and left at room temperature. After 24 hours when most of the solvent had vaporized, the polymer films were removed from the bath using milli-Q water. The formed polymer films were then placed into a vacuum chamber with initial pressure of $10^{-3} \mathrm{~Pa}$ and temperature of $25^{\circ} \mathrm{C}$ for 24 hours to remove the residual solvent.

The PLLA films were treated on the custom made magnetron device (Fig. 1) developed in Dr. Tverdokhlebov's lab. The magnetron has an elongated electrode that is placed horizontally into the vacuum chamber and was used with a target that is made of the hydroxyapatite powder $\left(\mathrm{Ca}_{5}\left(\mathrm{PO}_{4}\right)_{3}(\mathrm{OH})\right)$ with the size of $330 \times 120 \times 6 \mathrm{~mm}$. The device has $\mathrm{RF}$ generator with a maximum power of $4 \mathrm{~kW}$ and an operating frequency of $13.56 \mathrm{MHz}$. For treatment of PLLA films specific RF power was set to $5 \mathrm{~W} / \mathrm{cm}^{2}$ to maintain electron density within $10^{9}-10^{10} \mathrm{pp} \mathrm{cm}^{3}$ range and target-to-substrate distance was extended to $16 \mathrm{~cm}$ to facilitate enhanced coagulation of elemental plasma and target species under collision plasma conditions. Treatment duration was 30,60 and $150 \mathrm{sec}$. 


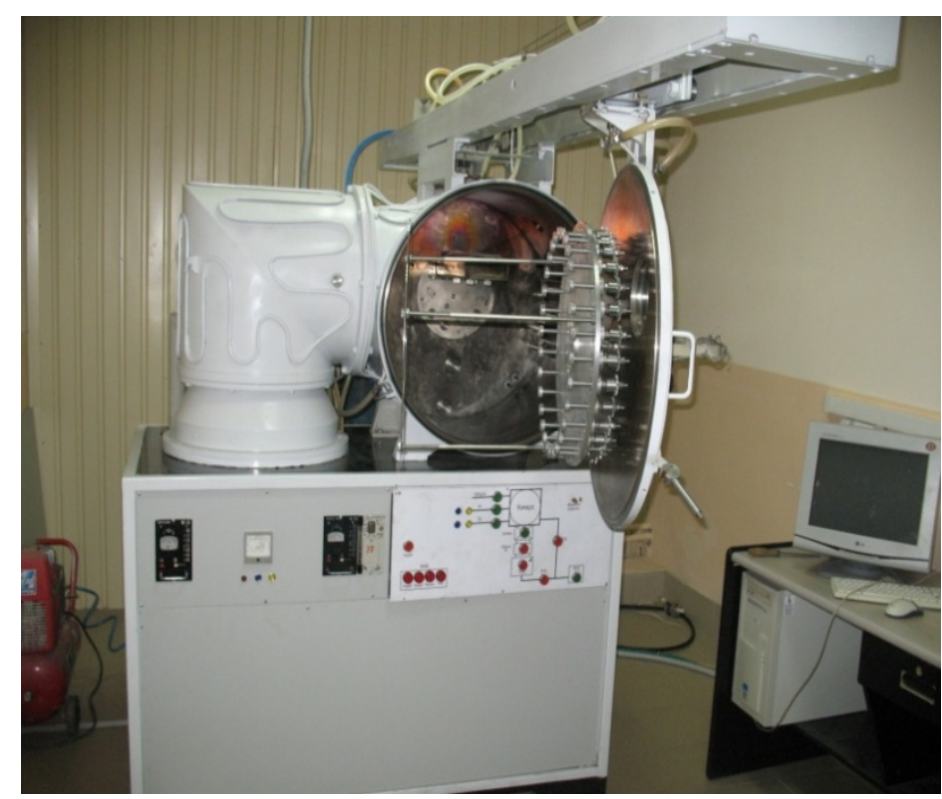

(a)

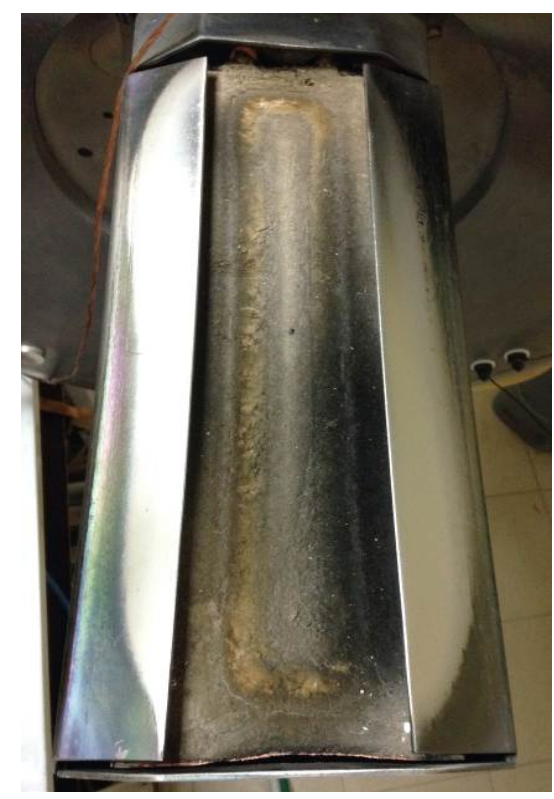

(b)

Fig. 1. RFMS device (a) and its elongated electrode (b).

High resolution atomic force microscopy (AFM) was used to investigate the surface morphology of samples. AFM microscope Solver-HV with cantilevers NSG11 (NT-MDT, Russia) was used. Processing of the images was performed using Gwyddion 2.25 software.

The chemical structure of the samples was studied using the Attenuated total reflectance Fourier transform infrared (FTIR) spectroscopy, using a Nicolet 6700 system (Thermo Scientific, USA) in the range of 800 to $2000 \mathrm{~cm}^{-1}$ with a resolution of $4 \mathrm{~cm}^{-1}$.

The investigation of the crystal structure of the samples was conducted by X-ray diffraction (XRD) analysis using a Shimadzu XRD 6000 diffractometer. The samples were irradiated with monochromatic $C u K \alpha$ radiation $(\lambda=1.54 \AA$ ) produced using the accelerating voltage of $40 \mathrm{kV}$ and the beam current of $30 \mathrm{~mA}$. The scanning angle range, scanning step size and signal collection time were $6-35^{\circ}, 0.02^{\circ}$ and 1.5 s respectively. The average size of the crystals $\left(l_{c}\right)$ of the samples was calculated using the Debye-Scherrer equation:

$$
l_{c}=\frac{k \lambda}{\cos \theta \sqrt{\beta^{2}-\beta_{r}^{2}}},
$$

where $\lambda$ is the wavelength of the incident radiation, $\beta$ the width of the reflection at half height, $\beta_{r}$ is the broadening reflex of the apparatus, $\theta$ is the angle of diffraction and $k=0.9$.

X-ray fluorescence (XRF) analysis using spectrometer Shimadzu XRF 1800 was used to investigate the elemental composition of the samples. The accelerating voltage, scanning speed 
and scanning step were set to $40 \mathrm{kV}, 8^{\circ} / \mathrm{min}$ and $0.1^{\circ}$ respectively. Studies were conducted via a carbon $(\mathrm{C})$, oxygen $(\mathrm{O})$, calcium $(\mathrm{Ca})$, phosphorus $(\mathrm{P})$ and chlorine $(\mathrm{Cl})$ channels.

Multipotent mesenchymal stromal cells from bone marrow (BM MMSCs) at second passage were used for cell adhesion investigation and were obtained from the Bank of Stem Cells Ltd (Tomsk, Russia). Efficiency of the cell adhesion to the modified surface was studied using fluorescent microscopy method with the inverted microscope Axio Observer Z1 (Carl Zeiss). The efficiency was evaluated by measuring the number of adherent cells visible in ten fields of view of the microscope, which were averaged and normalised to $1 \mathrm{~mm}^{2}$.

Preliminary estimation of the viability of these cells was performed by combined staining with Annexin V (BD Biosciences, USA), labelled with phycoerythrin (PE, BD Biosciences, USA) in combination with the 7-amino actinomycin D (7-AAD, BD Biosciences, USA). Phenotypic analysis of obtained cells was performed with monoclonal antibodies (anti-CD90 conjugated with Fluorescein Isothyocyanate and anti-CD45 conjugated with PE) on cytometer FACS Calibur (Becton Dickinson, USA).

Films were cut as disks with area of $1.8 \mathrm{~cm}^{2}$ for the BM MMSCs cultivation. Discs were seeded with MMSCs, placed on to 24-set culture plate (concentration of $2.5 \times 10^{5}$ cells per set) and cultivated for 5 days. Cells cultivation was carried out in culture medium DMEM (Gibco, USA) containing $1 \%$ of HEPES buffer, $10 \%$ of foetal bovine serum, $1 \%$ of L-glutamine, $100 \mathrm{U} / \mathrm{ml}$ of penicillin, $0.1 \mu \mathrm{g} / \mathrm{ml}$ of streptomycin, $0.1 \mu \mathrm{g} / \mathrm{ml}$ of amphotericin B (Sigma Aldrich, USA) at $37{ }^{\circ} \mathrm{C}$ and $5 \%$ of $\mathrm{CO}_{2}$.

The study of cellular viability on the modified surface was carried out with FCM technology on cytometer FACS Calibur (Becton Dickinson, USA). Cells were detached from the film surface by using a $0.5 \%$ solution of trypsin-EDTA (Sigma Aldrich), centrifuged for $10 \mathrm{~min}$ at $716 \mathrm{~g}$, resuspended in $1 \mathrm{ml}$ of the culture medium and stained with Annexin-V, labeled $\mathrm{PE}$, in combination with 7-AAD (BD Biosciences). Samples with non-modified PLLA surface were used as control.

The data was analyzed using the methods of statistical description and statistical hypothesis testing available in the standard software package Statistica (version 6.0). For the analysis of the data the hypothesis of normal distribution (Kolmogorov-Smirnov test) was used. In the case of the normal distribution the statistical significance was evaluated using the Student's $t$-test. When analyzed parameters had abnormal distribution, the estimation of the accuracy differences was determined using non-parametric criteria. To evaluate the accuracy of differences of three and 
more indicators within the same group the criterion of Friedman was used. In case of pairwise comparisons Wilcoxon test with Bonferroni correction was applied. Data was presented as a median and 25th and 75th percentiles (Me (25\%, 75\%)). The significance level was at least 95\% $(p<0.05)$ for all types of the statistical analysis.

\section{Results and discussion}

Fig. 2 shows high resolution AFM images of the surface of the investigated samples for different plasma treatment times. The AFM study of the surface shows the change in morphology and the increase in PLLA surface roughness with increasing the plasma treatment time. Specifically, Fig. 2(a)-(b) shows that non-modified PLLA surface has no significant cavities and protrusions. Fig. 2(c)-(d) shows that after the interaction with plasma for 30 seconds a significant number of round formations with an average diameter of $88 \pm 22 \mathrm{~nm}$ appears on the surface. Fig.2(e)-(f) shows that with increasing the treatment time to 60 seconds the formations' diameter increases to $240 \pm 40 \mathrm{~nm}$ and smaller formations with an average diameter of $35 \pm 4 \mathrm{~nm}$ are apparent on the surface of the large ones. At 150 seconds of the plasma treatment (Fig. 2 (g)-(h)) the surface of PLLA has a brain-like appearance with a higher surface roughness. 


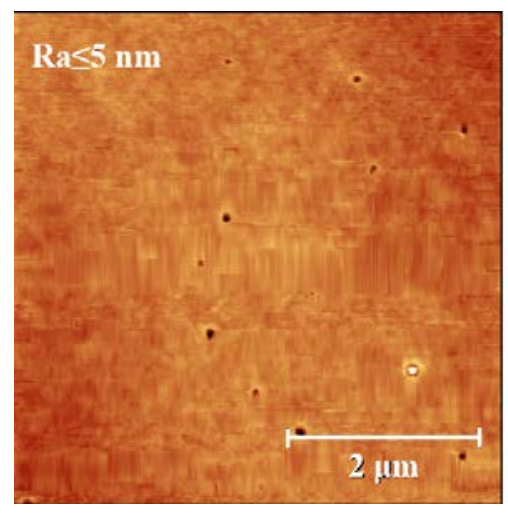

(a)

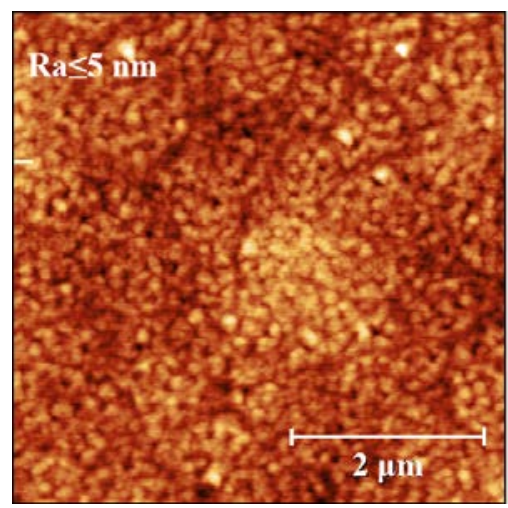

(c)

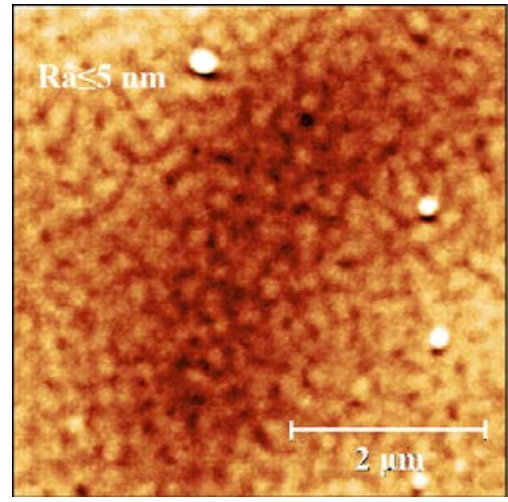

(e)

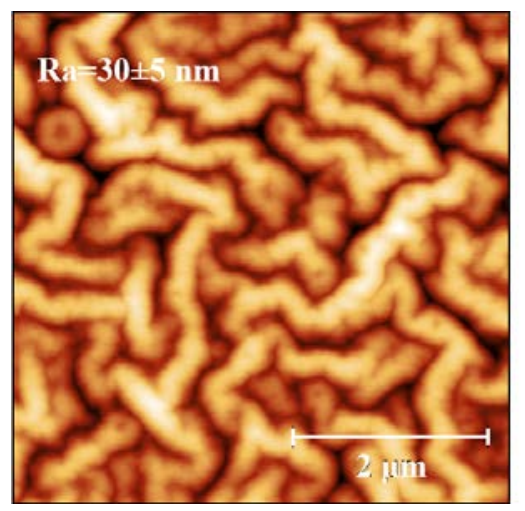

(g)
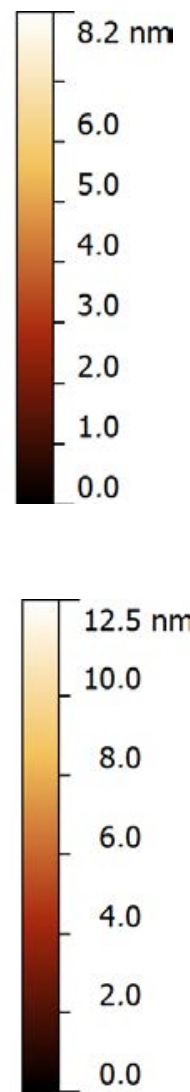

0.0
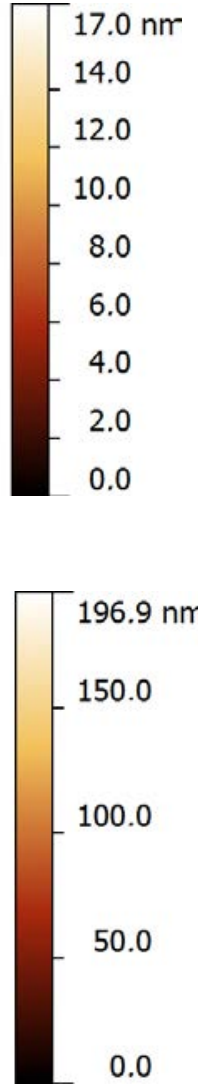

Fig. 2. AFM images of the PLLA surface at different plasma treatment times: $0 \mathrm{sec}$ (non-modified) (a)-(b), $30 \mathrm{sec}(\mathrm{c})-(\mathrm{d}), 60 \mathrm{sec}(\mathrm{e})-(\mathrm{f})$ and $150 \mathrm{sec}$ (g)-(h).

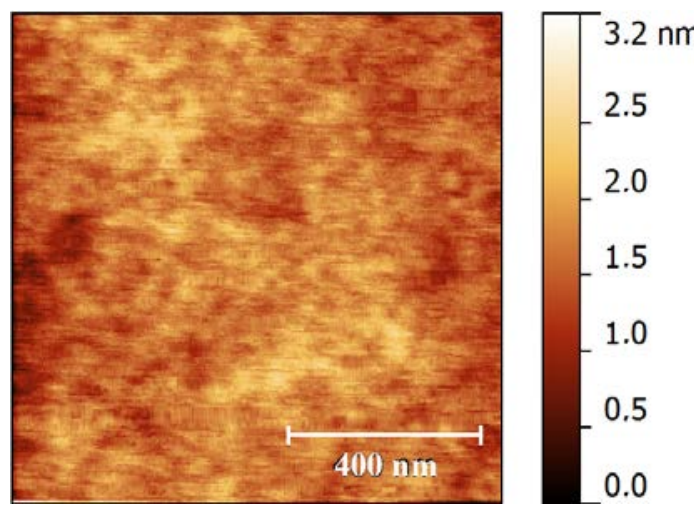

(b)

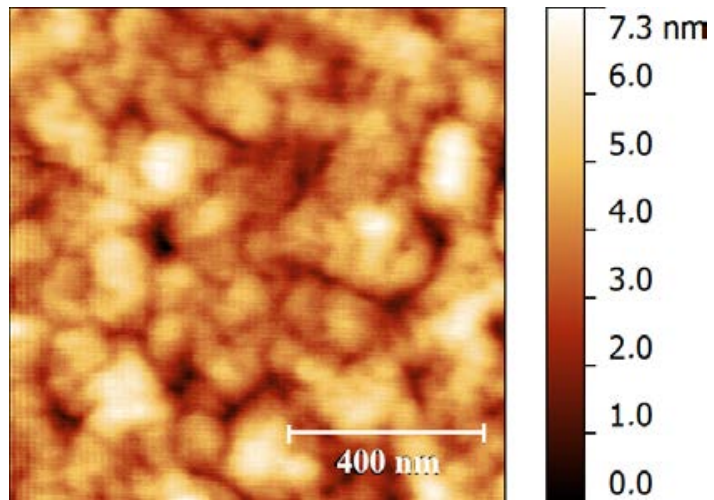

(d)

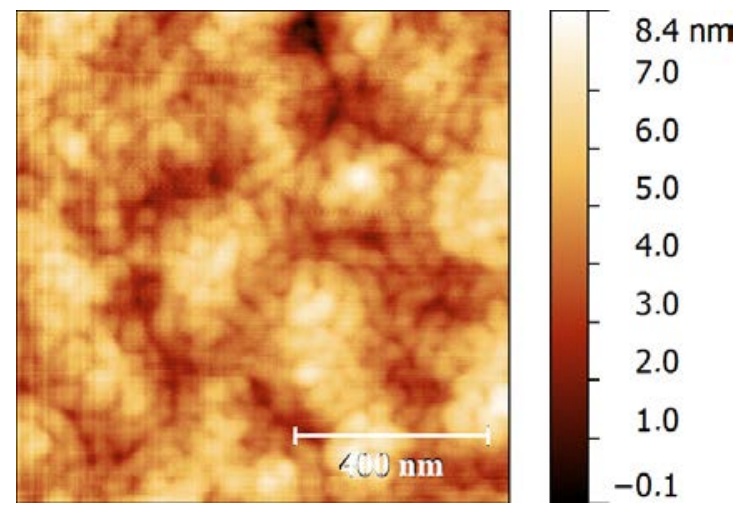

(f)

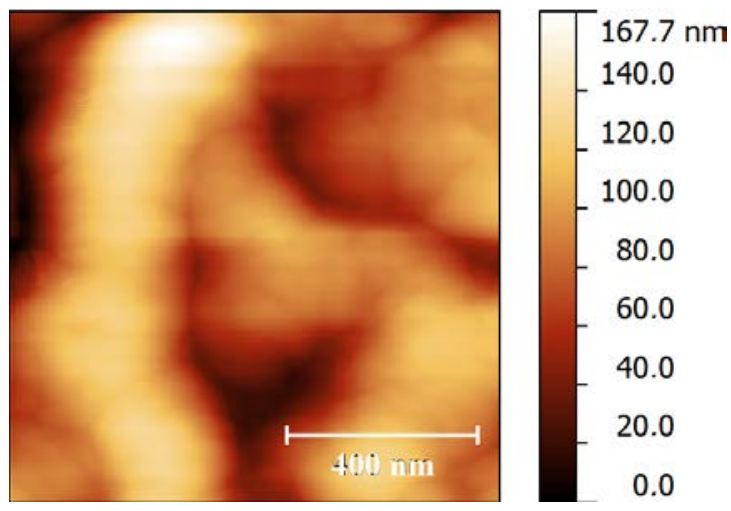

(h) 
There are two possible mechanisms responsible for the formation of the highly rough surface. The first "chemical" one is due to the PLLA degradation processes. It is known that the PLLA structure is a heterogeneous system consisting of ordered crystalline and amorphous parts. Amorphous regions located between the crystalline ones bind them together ensuring the structural integrity of the polymer system [27]. During the interaction of the PLLA surface with high-energy plasma particles (electrons, $\mathrm{Ar}^{+}$, ions of sputtered target $\left(\mathrm{CaO}^{+}, \mathrm{Ca}^{2+}, \mathrm{PHO}^{+}, \mathrm{PO}^{+}\right.$, $\left.\mathrm{P}^{+},\left(\mathrm{PO}_{4}^{-}\right)_{3}\right)$ amorphous regions are less stable than crystalline. As a result, there is a destruction of predominantly amorphous regions of the polymer under the plasma influence. Degradation products are removed by the vacuum system, resulting to a selective etching of the surface. Thus, the short time plasma treatment of the polymer leads to the formation of highly rough surface primarily due to the degradation processes of amorphous regions. The second "physical" mechanism is mostly connected with the processes of PLLA crystallization. High-energy plasma particles lose their kinetic energy by damping on PLLA surface which causes intense surface heating. It is known that amorphous regions of PLLA are able to transform into the crystalline state as a result of a thermal impact, and this process rate is proportional to the crystallization temperature [28]. The effect of the increasing PLLA crystallinity degree under the temperature influence was described for isothermal crystallization of films [29] and nonwoven materials [30]. Since the formed polymer crystal occupies a smaller volume than the amorphous region the surface "contraction" occurs, which leads to the formation of the rough surface.

Previous studies of PLLA crystallization processes with the help of IR spectroscopy revealed the bands in the spectra sensitive to changes in the crystal structure of PLLA macromolecules (Table 1). Therefore, IR spectra can be used to estimate changes in PLLA crystal structure.

\section{Table 1}

Relevant IR bands associated with different phases of PLLA (modified from [30]).

\begin{tabular}{|c|c|c|}
\hline IR frequency $\left(\mathrm{cm}^{-1}\right)$ & Crystalline form & Ref. № \\
\hline 860 & Amorphous & {$[31]$} \\
\hline 871 & $\alpha$ & {$[32]$} \\
\hline 908 & $\beta$ & {$[33]$} \\
\hline 921 & $\alpha$ & {$[33]$} \\
\hline 955 & Amorphous & {$[34]$} \\
\hline 1268 & Amorphous & {$[34]$} \\
\hline 1358 & Semicrystalline & {$[35]$} \\
\hline 1749 & $\alpha$ & {$[36]$} \\
\hline
\end{tabular}

Fig. 3 shows the IR spectra of the samples where we can observe an increase in the degree of PLLA crystallinity with the increasing of the plasma treatment time. Thus, there is an increase of 
the $1749 \mathrm{~cm}^{-1}$ band intensity in the $1810-1710 \mathrm{~cm}^{-1}$ region (the $\mathrm{C}=\mathrm{O}$ stretching band region). There is an increase in the intensity of the band $1358 \mathrm{~cm}^{-1}$ with simultaneous decrease in the intensity of the band $1268 \mathrm{~cm}^{-1}$ in the region $1200-1500 \mathrm{~cm}^{-1}$ (the $\mathrm{CH}_{3}$, $\mathrm{CH}$ bending, and $\mathrm{C}-\mathrm{O}-\mathrm{C}$ stretching band region). The region of $840-960 \mathrm{~cm}^{-1}$ (the skeletal stretching and $\mathrm{CH}_{3}$ rocking band region) has an increase in $921 \mathrm{~cm}^{-1}$ band intensity and a decrease at $955 \mathrm{~cm}^{-1}$. There is band shift from $860 \mathrm{~cm}^{-1}$ to $871 \mathrm{~cm}^{-1}$. The absence of the band at $908 \mathrm{~cm}^{-1}$ characteristic for PLLA in $\beta$-crystal form is noteworthy. It is known that the $\alpha$-crystal modification of PLLA is easily formed from the melt $[37,38]$, therefore, the increase in the intensity of the absorption bands characteristic for the $\alpha$-crystal structure of PLLA is an indication that the process of PLLA crystallization is caused by heating of the polymer surface as a result of the interaction with high-energy plasma particles.

PLLA film is in mostly amorphous state before the treatment which is evidenced by a vast halo observed at $15-25^{\circ}$ (Fig. 4). The mostly amorphous state is due to a low crystallization rate of the PLLA solution at room temperature [33]. Fig. 4 shows changes of the reflections for treated films. The increase of the reflection (110/200) intensity at $16.5^{\circ}$ is connected with the growth of the polymer crystal in the direction (110/200). The crystal size for 30 sec treatment is less than $10 \mathrm{~nm}$, for 60 and $150 \mathrm{sec}$ treatment times the crystal size is $16.6 \pm 0.4$ and $21.1 \pm 0.3 \mathrm{~nm}$ respectively. There is a shift of several reflexes: $(110 / 200)$ to the region of $16.6-16.7^{\circ}$, reflex (203) from 18.6 to $18.8^{\circ}$. There is an increase in the intensity of minor, but noteworthy reflections at 12.1 (004/103), 14.7 (010) and 22.3 (015).

The obtained dependences are the evidence of PLLA crystallization mechanism "amorphous state $\rightarrow \alpha^{\prime} \rightarrow \alpha$ " described in [28, 39]. It is known that this crystallization mechanism is observed in the temperature range $110-170{ }^{\circ} \mathrm{C}$. Transition "amorphous state $\rightarrow \alpha^{\prime} \rightarrow \alpha^{\prime}$ detected by XRD is an indication of the significant increase in the PLLA surface temperature due to the interaction with plasma. Thus, PLLA crystallization processes in the formation of the surface relief play an important role when the time of the interaction between PLLA surface and plasma is significant. The formation of the brain-like appearance after the surface treatment for $150 \mathrm{sec}$ (Fig. 2(g)-(h)) is caused by the processes of the fusion of $\alpha^{\prime}$ type polymer crystals (formed on the surface during the short time plasma treatment) and the formation of highly ordered $\alpha$ type crystals formed because of the surface heating.

Fig. 5 shows the typical fluorescence spectra of the investigated samples for the following elements: carbon, oxygen, chlorine, calcium and phosphorus. 


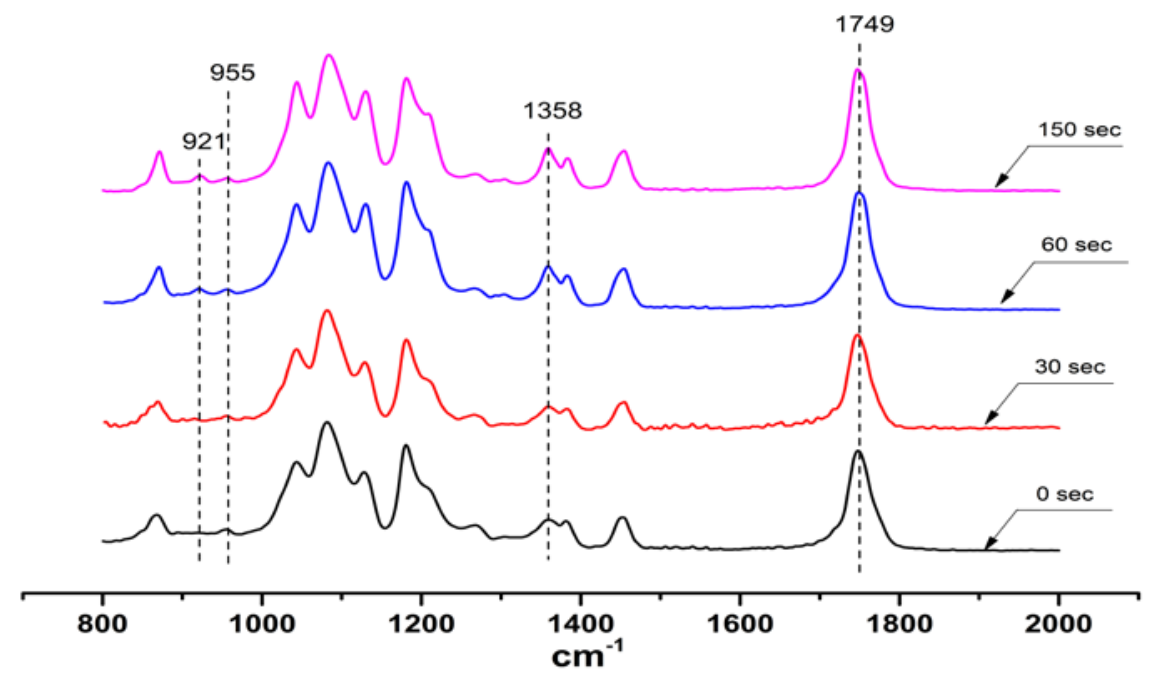

(a)

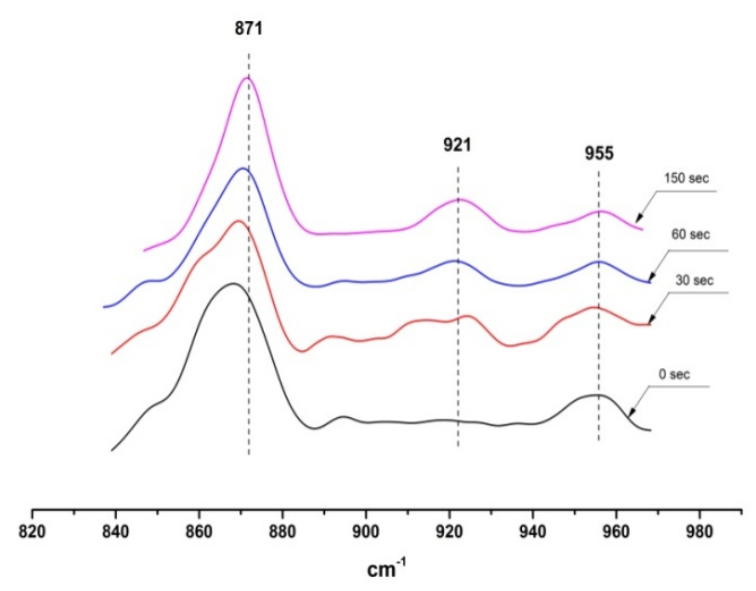

(b)



(c)

Fig. 3. FTIR results of the investigated samples: 800-2000 (a), 820-980 (b) and 1200-1500 cm ${ }^{-1}$ (c) regions. 


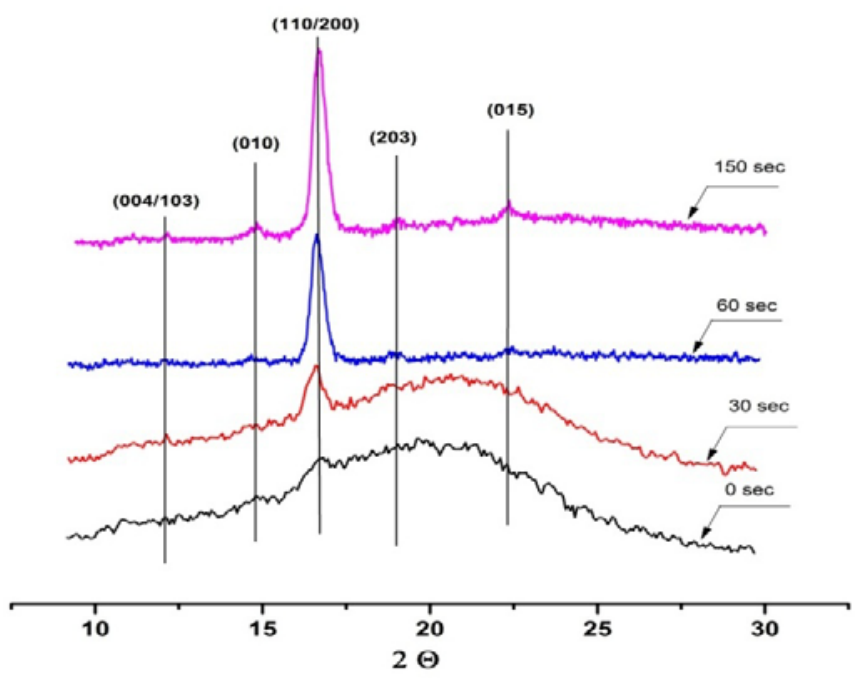

Fig. 4. XRD results of the investigated samples.

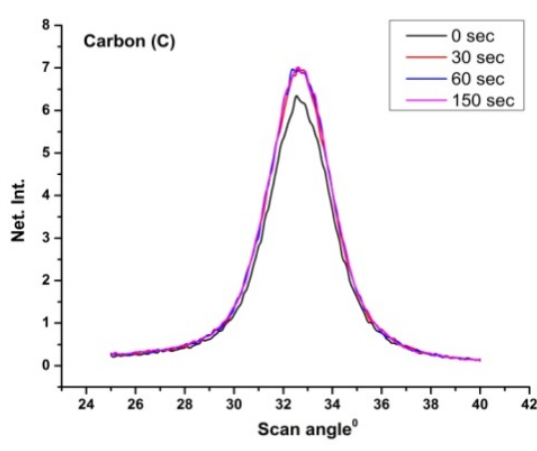

(a)

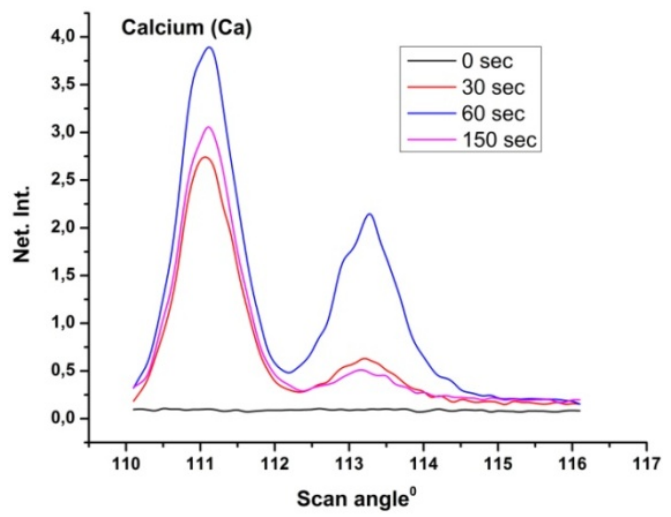

(d)

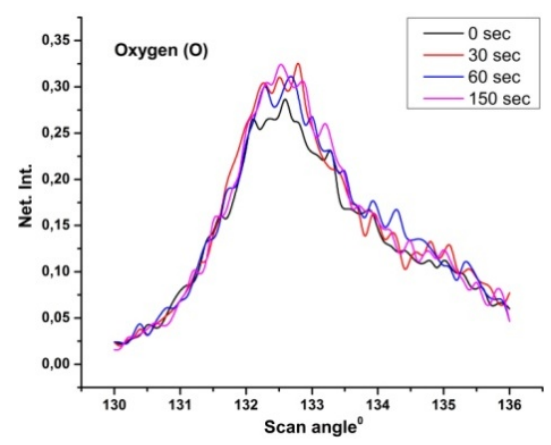

(b)

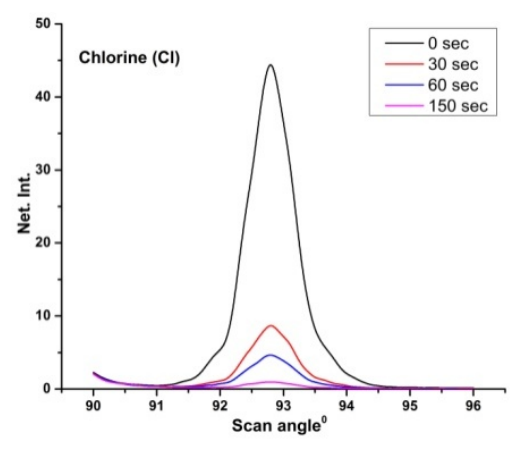

(c)

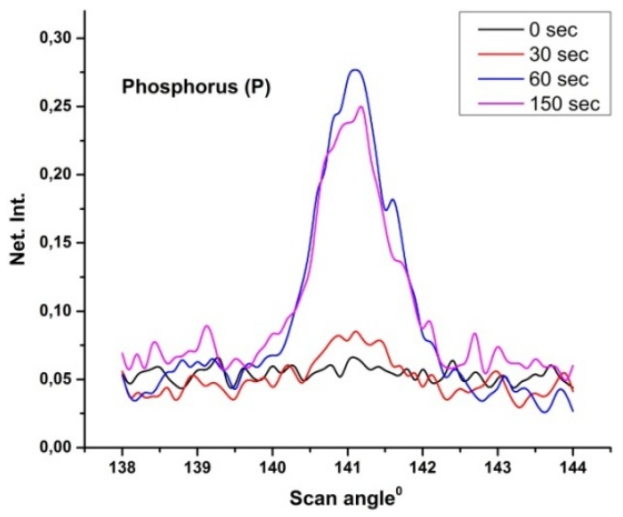

(e)

Fig. 5. Fluorescent spectra of the investigated samples for the following elements. Channels of C (a), O (b), Cl (c), Ca (d) and P (e). 
Results of semi-quantitative elemental analysis of the investigated samples before and after the plasma treatment are shown in Table 2. Increasing the treatment time to 150 seconds gives the decrease of chlorine $(\mathrm{Cl})$ content by more than 18 times in comparison with non-modified film. This phenomenon may be observed due to the increase of the free surface on the films after treatment which allows an easier diffusion of dichloromethane residual from the bulk to the surface of the film and its subsequent removal into the vacuum system of the sputtering facility. We believe that the slight decrease of the carbon content (on average 2.5\%) with the simultaneous increase of the oxygen content (on average 6\%) can be explained by the formation of $\mathrm{C}-\mathrm{O}$ and $\mathrm{O}-\mathrm{C}=\mathrm{O}$ functional groups with additional oxygen ions from the sputtered hydroxyapatite target.

\section{Table 2}

Semi-quantitative analysis of elemental composition of the investigated samples.

\begin{tabular}{|c|c|c|c|c|c|}
\hline \multirow{2}{*}{$\begin{array}{l}\text { Treatment } \\
\text { time, sec }\end{array}$} & \multicolumn{5}{|c|}{ Content the element, wt.\% } \\
\hline & $\mathrm{Ca}$ & $\mathrm{P}$ & $\mathrm{C}$ & $\mathrm{O}$ & $\mathrm{Cl}$ \\
\hline 0 & $<0.01$ & $<0.01$ & $63.22 \pm 2.24$ & $32.23 \pm 1.74$ & $4.54 \pm 1.22$ \\
\hline 30 & $0.02 \pm 0.02$ & $<0.01$ & $62.88 \pm 2.68$ & $35.92 \pm 2.22$ & $1.17 \pm 0.43$ \\
\hline 60 & $0.07 \pm 0.04$ & $\approx 0.01$ & $61.73 \pm 2.12$ & $37.59 \pm 1.83$ & $0.61 \pm 0.32$ \\
\hline 150 & $<0.02$ & $<0.01$ & $60.58 \pm 2.54$ & $39.17 \pm 1.68$ & $0.25 \pm 0.24$ \\
\hline
\end{tabular}

The content of calcium and phosphorus in PLLA films reached maximum value at 60 sec plasma treatment time and then decreased for $150 \mathrm{sec}$ treatment. When the treatment time is relatively small (e.g. $30 \mathrm{sec}$ ), bombardment of the PLLA surface by ions of the sputtered target results in formation of a thin amorphous ((bio-)ceramic/(bio-)resorbable polymer layer. For longer treatment time (60-150 sec) PLLA surface heats up, leading to the increase of the thermochemical degradation rate of PLLA surface. Degradation also happens because the treated surface is bombarded by $\mathrm{O}^{-2}$ sputtered from the hydroxyapatite target. As a result, the surface is enriched with PLLA degradation products (low molecular weight polymers, oligomers, etc.). As these products are weakly bounded to the surface, this leads to their removal by the vacuum system installed on the sputtering facility together with ions of calcium and phosphorus.

Table 3 shows the results of the studies of the effect of PLLA surface treatment on BM MMSCs. Fig. 6 shows the images of fluorescently labelled cells on the investigated PLLA surfaces. 


\section{Table 3}

The results of biological tests of the investigated samples.

\begin{tabular}{|l|l|l|l|l|l|}
\hline \multirow{2}{*}{$\begin{array}{l}\text { Treatment } \\
\text { time, sec }\end{array}$} & $\begin{array}{l}\text { Amount of BM } \\
\text { MMSCs } \\
\text { on 1 mm of the } \\
\text { surface, } \\
\text { Me }(25 \% ; 75 \%)\end{array}$ & \multicolumn{4}{|l|}{ Relative amount of BM MMSCs, (Me (25\%; 75\%)) } \\
\cline { 3 - 6 } & $\begin{array}{l}\text { Viable, \% } \\
\text { (12 }\end{array}$ & $\begin{array}{l}\text { Early } \\
\text { apoptosis, \% }\end{array}$ & $\begin{array}{l}\text { Late } \\
\text { apoptosis, \% }\end{array}$ & Necrosis, \% \\
\hline 0 & $(105 ; 174)$ & 44.9 & 11.7 & 17.0 & 26.3 \\
\hline 30 & 482 & 66.1 & 29.7 & 3.12 & $(16.9 ; 35.7)$ \\
\hline 60 & $(370 ; 614)$ & $(60.5 ; 71.8)$ & $(26.6 ; 32.8)$ & $(1.10 ; 5.14)$ & $(0.53 ; 1.47)$ \\
\hline 150 & 707 & 71.0 & 24.4 & 1.95 & 2.65 \\
& $(683 ; 829)$ & $(62.7 ; 79.2)$ & $(14.0 ; 34.9)$ & $(1.07 ; 2.83)$ & $(1.37 ; 3.93)$ \\
\hline & 715 & 71.7 & 24.4 & 2.07 & 1.79 \\
& $(654 ; 791)$ & $(68.0 ; 74.6)$ & $(19.8 ; 29.8)$ & $(1.07 ; 3.07)$ & $(1.10 ; 2.47)$ \\
\hline
\end{tabular}



(a)



(c)

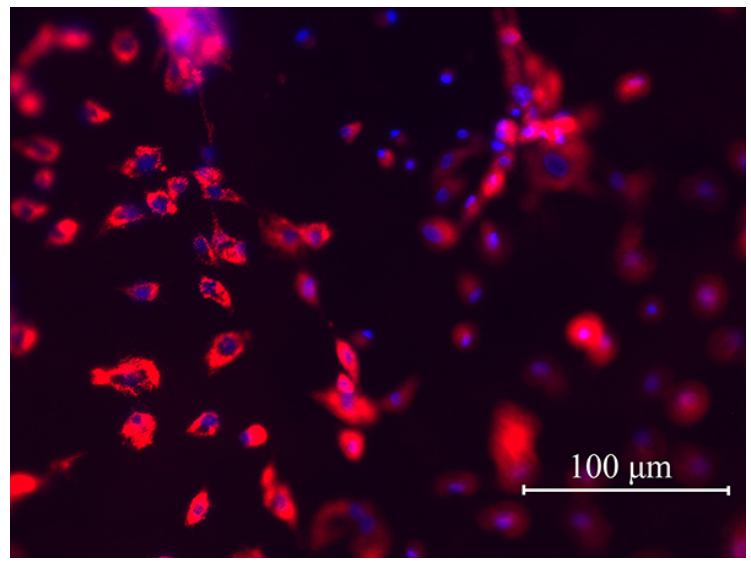

(b)

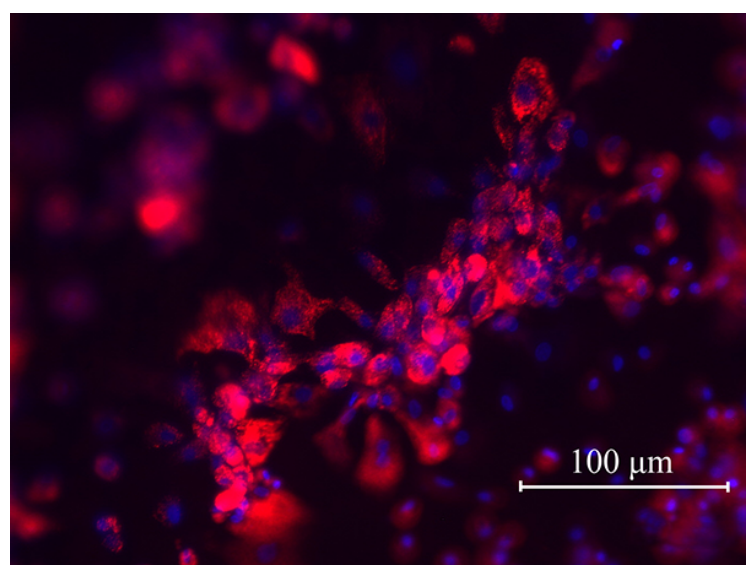

(d)

Fig. 6. Images of fluorescent-labelled cells on the investigated surfaces: non-modified (a), after treatment for $30 \mathrm{sec}(\mathrm{b}), 60 \mathrm{sec}(\mathrm{c})$ and $150 \mathrm{sec}(\mathrm{d})$. 
The significant increase in cell adhesion to modified PLLA surface is evident after 5 days of BM MMSCs cultivation (Table 3, Fig. 6). The total number of adherent cells on the surface modified for 30 sec was 4.3 times higher compared to the non-modified surface. The modification for 60 and $150 \mathrm{sec}$ has given similar significant increase (about 6.3 times) in the adherent cells compared to non-modified surface ( $\mathrm{p}<0.05$ ). Thus, there was no significant difference between the amount of BM MMSCs on the PLLA films treated for 60 and 150 sec.

The analysis of BM MMSCs culture on all the investigated surfaces using FCM demonstrated that $98.5 \%$ are CD90+ CD45- cells. Therefore, the surface properties do not cause the change of cellular phenotype during 5 days of BM MMSCs cultivation.

The investigation of cell viability of BM MMSCs, cultivated for 5 days (Table 3), demonstrated that the surface modification increased the number of viable BM MMSCs by 1.5-1.6 times $(\mathrm{p}<0.05)$ compared to the non-modified PLLA surface. Early apoptosis of BM MMSCs was less frequent for non-modified surface in comparison with plasma treated one (2.5, 2.1 and 2.1 times less for 30, 60 and 150 sec treatments, respectively; p <0.05). However, a significantly lower percentage of late apoptotic and necrotic cells was found on the modified PLLA surface comparing with non-modified ones. This observation raises a question regarding the genesis of the high proportion of BM MMSCs early apoptosis. It is known that cell activation level can have a significant effect on the increase of early apoptosis. The cell activation level promotes the emergence of phosphatidylserine molecules on surface of cells, for example as a result of active cell proliferation. This emergence may result in the increase of phosphatidylserine binding with Annexin-V [40]. Thus, the data for early apoptotic BM MMSCs suggests two possible mechanisms of early apoptosis: the first mechanism is the beginning of programmed cell death, whereas the second one is the active cell proliferation.

The rate of late apoptosis decreased by about 6 to 9 times on the modified surfaces comparing to non-modified surfaces, which indicates more favorable conditions for BM MMSCs cultivation on modified PLLA surfaces. The total rate of cell necrosis was 10 to 26 times lower on modified PLLA surface comparing to non-modified samples surface $(\mathrm{p}<0.05)$.

\section{Conclusions}

It was shown that the RFMS plasma treatment with the solid hydroxyapatite target sputtering increases the biocompatibility of PLLA surface by stimulating processes of attachment and differentiation of MMSC pool. The FCM method revealed that the plasma treatment does not cause an adverse cellular response (apoptosis, necrosis). This surface modification can therefore 
enhance PLLA usability as a biomaterial. It is hypothesised that the increased surface roughness of PLLA, which was demonstrated with AFM studies, plays a major role in the increased biocompatibility. FTIR, XRD studies of the surface demonstrated that the formation of the rough surface morphology was caused by the processes of PLLA crystallization. XRF studies also have confirmed that PLLA surface was significantly enriched by calcium and phosphorus from the hydroxyapatite target at longer treatment times. This enrichment can also contribute to improvements in the biocompatibility of the PLLA surface.

\section{Acknowledgments}

The authors would like to thank V. Novikov for conducting AFM studies. This work was financially supported by the Russian Foundation for Basic Research project \#13-08-98052, Federal Target Program (state contract \# 14.577.21.0036).

\section{References}

[1] L.S. Nair, C.T. Laurencin, Biodegradable polymers as biomaterials, Prog Polym Sci, 32 (2007) 762-798. [2] H.Y. Tian, Z.H. Tang, X.L. Zhuang, X.S. Chen, X.B. Jing, Biodegradable synthetic polymers: Preparation, functionalization and biomedical application, Prog Polym Sci, 37 (2012) 237-280.

[3] I. Armentano, M. Dottori, E. Fortunati, S. Mattioli, J.M. Kenny, Biodegradable polymer matrix nanocomposites for tissue engineering: A review, Polymer Degradation and Stability, 95 (2010) 21262146.

[4] S. Yoshida, K. Hagiwara, T. Hasebe, A. Hotta, Surface modification of polymers by plasma treatments for the enhancement of biocompatibility and controlled drug release, Surf Coat Tech, 233 (2013) 99-107.

[5] S. Bauer, P. Schmuki, K. von der Mark, J. Park, Engineering biocompatible implant surfaces Part I: Materials and surfaces, Prog Mater Sci, 58 (2013) 261-326.

[6] K. von der Mark, J. Park, Engineering biocompatible implant surfaces Part II: Cellular recognition of biomaterial surfaces: Lessons from cell-matrix interactions, Prog Mater Sci, 58 (2013) 327-381.

[7] H. Chen, L. Yuan, W. Song, Z. Wu, D. Li, Biocompatible polymer materials: Role of protein-surface interactions, Prog Polym Sci, 33 (2008) 1059-1087.

[8] R.M. Rasal, A.V. Janorkar, D.E. Hirt, Poly(lactic acid) modifications, Prog Polym Sci, 35 (2010) 338-356.

[9] F. Poncin-Epaillard, O. Shavdina, D. Debarnot, Elaboration and surface modification of structured poly(L-lactic acid) thin film on various substrates, Mat Sci Eng C-Mater, 33 (2013) 2526-2533.

[10] K.S. Siow, L. Britcher, S. Kumar, H.J. Griesser, Plasma methods for the generation of chemically reactive surfaces for biomolecule immobilization and cell colonization - A review, Plasma Process Polym, 3 (2006) 392-418.

[11] R. Morent, N. De Geyter, T. Desmet, P. Dubruel, C. Leys, Plasma Surface Modification of Biodegradable Polymers: A Review, Plasma Process Polym, 8 (2011) 171-190.

[12] A.R. Boyd, C. O'Kane, P. O'Hare, G.A. Burke, B.J. Meenan, The influence of target stoichiometry on early cell adhesion of co-sputtered calcium-phosphate surfaces, J Mater Sci-Mater M, 24 (2013) 28452861.

[13] V.F. Pichugin, R.A. Surmenev, E.V. Shesterikov, M.A. Ryabtseva, E.V. Eshenko, S.I. Tverdokhlebov, O. Prymak, M. Epple, The preparation of calcium phosphate coatings on titanium and nickel-titanium by rfmagnetron-sputtered deposition: Composition, structure and micromechanical properties, Surf Coat Tech, 202 (2008) 3913-3920. 
[14] S.I. Tverdokhlebov, E.N. Bolbasov, E.V. Shesterikov, A.I. Malchikhina, V.A. Novikov, Y.G. Anissimov, Research of the surface properties of the thermoplastic copolymer of vinilidene fluoride and tetrafluoroethylene modified with radio-frequency magnetron sputtering for medical application, Appl Surf Sci, 263 (2012) 187-194.

[15] J.E.G. Hulshoff, K. Vandijk, J.P.C.M. Vanderwaerden, J.G.C. Wolke, L.A. Ginsel, J.A. Jansen, Biological Evaluation of the Effect of Magnetron-Sputtered Ca/P Coatings on Osteoblast-Like Cells in-Vitro, J Biomed Mater Res, 29 (1995) 967-975.

[16] B. Feddes, A.M. Vredenberg, J.G.C. Wolke, J.A. Jansen, Bulk composition of r.f. magnetron sputter deposited calcium phosphate coatings on different substrates (polyethylene, polytetrafluoroethylene, silicon), Surf Coat Tech, 185 (2004) 346-355.

[17] D.V. Shtansky, A.S. Grigoryan, A.K. Toporkova, A.V. Arkhipov, A.N. Sheveyko, P.V. KiryukhantsevKorneev, Modification of polytetrafluoroethylene implants by depositing TiCaPCON films with and without stem cells, Surf Coat Tech, 206 (2011) 1188-1195.

[18] B. Feddes, J.G.C. Wolke, A.M. Vredenberg, J.A. Jansen, Initial deposition of calcium phosphate ceramic on polyethylene and polydimethylsiloxane by $\mathrm{rf}$ magnetron sputtering deposition: the interface chemistry, Biomaterials, 25 (2004) 633-639.

[19] M.E. Konischev, O.S. Kuzmin, A.A. Pustovalova, N.S. Morozova, K.E. Evdokimov, R.A. Surmenev, V.F. Pichugin, M. Epple, Structure and Properties of Ti-O-N Coatings Produced by Reactive Magnetron Sputtering, Russ Phys J+, 56 (2014) 1144-1149.

[20] A.R. Boyd, C. O'Kane, B.J. Meenan, Control of calcium phosphate thin film stoichiometry using multi-target sputter deposition, Surf Coat Tech, 233 (2013) 131-139.

[21] T.Y. Ma, M.H. Choi, Optical and electrical properties of Mg-doped zinc tin oxide films prepared by radio frequency magnetron sputtering, Appl Surf Sci, 286 (2013) 131-136.

[22] A.Z.A. Djafer, N. Saoula, N. Madaoui, A. Zerizer, Deposition and characterization of titanium carbide thin films by magnetron sputtering using Ti and TiC targets, Appl Surf Sci, 312 (2014) 57-62.

[23] D.V. Shtansky, I.V. Batenina, P.V. Kiryukhantsev-Korneev, A.N. Sheveyko, K.A. Kuptsov, I.Y. Zhitnyak, N.Y. Anisimova, N.A. Gloushankova, Ag- and Cu-doped multifunctional bioactive nanostructured TiCaPCON films, Appl Surf Sci, 285 (2013) 331-343.

[24] G.H. Ryu, W.S. Yang, H.W. Roh, I.S. Lee, J.K. Kim, G.H. Lee, D.H. Lee, B.J. Park, M.S. Lee, J.C. Park, Plasma surface modification of poly(D,L-lactic-co-glycolic acid)(65/35) film for tissue engineering, Surf Coat Tech, 193 (2005) 60-64.

[25] B. Feddes, J.G.C. Wolke, W.P. Weinhold, A.M. Vredenberg, J.A. Jansen, Adhesion of calcium phosphate coatings on polyethylene (PE), polystyrene (PS), poly(tetrafluoroethylene) (PTFE), poly(dimethylsiloxane) (PDMS) and poly-L-lactic acid (PLLA), J Adhes Sci Technol, 18 (2004) 655-672.

[26] E.N. Bolbasov, M. Rybachuk, A.S. Golovkin, L.V. Antonova, E.V. Shesterikov, A.I. Malchikhina, V.A. Novikov, Y.G. Anissimov, S.I. Tverdokhlebov, Surface modification of poly(l-lactide) and polycaprolactone bioresorbable polymers using RF plasma discharge with sputter deposition of a hydroxyapatite target, Materials Letters, 132 (2014) 281-284.

[27] S. Saeidlou, M.A. Huneault, H.B. Li, C.B. Park, Poly(lactic acid) crystallization, Prog Polym Sci, 37 (2012) 1657-1677.

[28] M. Yasuniwa, K. Sakamo, Y. Ono, W. Kawahara, Melting behavior of poly(I-lactic acid): X-ray and DSC analyses of the melting process, Polymer, 49 (2008) 1943-1951.

[29] E. Lizundia, S. Petisco, J.-R. Sarasua, Phase-structure and mechanical properties of isothermally melt-and cold-crystallized poly (L-lactide), Journal of the Mechanical Behavior of Biomedical Materials, 17 (2013) 242-251.

[30] C. Ribeiro, V. Sencadas, C.M. Costa, J.L.G. Ribelles, S. Lanceros-Méndez, Tailoring the morphology and crystallinity of poly(L-lactide acid) electrospun membranes, Science and Technology of Advanced Materials, 12 (2011) 1-9.

[31] J. Zhang, H. Tsuji, I. Noda, Y. Ozaki, Weak Intermolecular Interactions during the Melt Crystallization of Poly(I-lactide) Investigated by Two-Dimensional Infrared Correlation Spectroscopy, The Journal of Physical Chemistry B, 108 (2004) 11514-11520. 
[32] N. Vasanthan, O. Ly, Effect of microstructure on hydrolytic degradation studies of poly (I-lactic acid) by FTIR spectroscopy and differential scanning calorimetry, Polymer Degradation and Stability, 94 (2009) 1364-1372.

[33] J. Zhang, H. Tsuji, I. Noda, Y. Ozaki, Structural Changes and Crystallization Dynamics of Poly(I-lactide) during the Cold-Crystallization Process Investigated by Infrared and Two-Dimensional Infrared Correlation Spectroscopy, Macromolecules, 37 (2004) 6433-6439.

[34] G. Kister, G. Cassanas, M. Vert, Effects of morphology, conformation and configuration on the IR and Raman spectra of various poly(lactic acid)s, Polymer, 39 (1998) 267-273.

[35] J. Zhang, Y. Duan, H. Sato, H. Tsuji, I. Noda, S. Yan, Y. Ozaki, Crystal Modifications and Thermal Behavior of Poly(I-lactic acid) Revealed by Infrared Spectroscopy, Macromolecules, 38 (2005) 8012-8021. [36] P. Pan, W. Kai, B. Zhu, T. Dong, Y. Inoue, Polymorphous Crystallization and Multiple Melting Behavior of Poly(I-lactide): Molecular Weight Dependence, Macromolecules, 40 (2007) 6898-6905.

[37] P. Pan, Z. Liang, B. Zhu, T. Dong, Y. Inoue, Blending Effects on Polymorphic Crystallization of Poly(Ilactide), Macromolecules, 42 (2009) 3374-3380.

[38] P. De Santis, A.J. Kovacs, Molecular conformation of poly(S-lactic acid), Biopolymers, 6 (1968) 299306.

[39] J. Zhang, K. Tashiro, H. Tsuji, A.J. Domb, Disorder-to-Order Phase Transition and Multiple Melting Behavior of Poly(l-lactide) Investigated by Simultaneous Measurements of WAXD and DSC, Macromolecules, 41 (2008) 1352-1357.

[40] K. Fischer, S. Voelkl, J. Berger, R. Andreesen, T. Pomorski, A. Mackensen, Antigen recognition induces phosphatidylserine exposure on the cell surface of human CD8+ T cells, Blood, 108 (2006) 40944101. 